\title{
Association of Serum Lipids with Outcomes in Hispanic Hemodialysis Patients of the West versus East Coasts of the United States
}

\author{
Hamid Moradi ${ }^{a}$ d Pouya Abhari ${ }^{a}$ Elani Streja ${ }^{a}$ Moti L. Kashyap ${ }^{b, e}$ \\ Gaurang Shah ${ }^{a, d}$ Daniel Gillen ${ }^{c}$ Madeleine V. Pahla Nosratola D. Vaziria \\ Kamyar Kalantar-Zadeh ${ }^{a}$ \\ ${ }^{a}$ Division of Nephrology and Hypertension, ${ }^{b}$ Department of Medicine, ${ }^{c}$ University of California Irvine School of \\ Medicine, Orange, Calif., ${ }^{d}$ Nephrology Section and ${ }^{~}$ Atherosclerosis Research Center, Veterans Affairs Healthcare System, \\ Long Beach, Calif., USA
}

\section{Key Words}

Lipids - Mortality · Hispanic · ESRD · Cardiovascular disease · Ethnicity $\cdot$ Race

\begin{abstract}
Background: Paradoxical associations exist between serum lipid levels and mortality in patients on maintenance hemodialysis (MHD) including those of Hispanic origin. However, there are significant racial and ethnic variations in patients of 'Hispanic' background. We hypothesized that clinically meaningful differences existed in the association between lipids and survival in Hispanic MHD patients on the West versus East Coast. Methods: We examined the survival impact of serum lipids in a 2-year cohort of 15,109 MHD patients of Hispanic origin being treated in California, Texas, representing the West versus New York, New Jersey and Florida representing the East Coast, using Cox models with various degrees of adjustments. Results: The association of serum total and HDL cholesterol with mortality follows a U-shaped pattern in Hispanic patients residing in the West. This is in contrast to Hispanic patients in the East Coast whose survival seems to improve with increasing total and $\mathrm{HDL}$ cholesterol levels. Elevated serum LDL levels in Hispanic patients on the West Coast are associated with a significant increase in mor-
\end{abstract}

tality, while this association is not observed in patients residing on the East Coast. Conclusions: Substantial differences exist in the association of serum lipids with mortality in MHD patients of Hispanic background depending on whether they reside on the West or East Coast of the United States. These geographical variances most likely reflect ethnic, racial and genetic distinctions, which are usually ignored. Future studies should take into account these critical variations in a population of patients who make up a significant portion of our society.

(c) 2015 S. Karger AG, Basel

\section{Introduction}

The number of patients with end-stage renal disease (ESRD) requiring maintenance dialysis in the United States currently stands at approximately 450,000 [1]. Despite many recent improvements in dialysis treatment and the adherence of patients and physicians to the quality measures set forth by guidelines, ESRD patients continue to experience an annual mortality rate of approximately $20 \%$, a rate worse than that of many cancers. While the causes of death in these patients are diverse, approximately half of all deaths in dialysis patients are directly attrib-

\section{KARGER 125}

() 2015 S. Karger AG, Base

0250-8095/15/0415-0284\$39.50/0

E-Mail karger@karger.com

www.karger.com/ajn
Kamyar Kalantar-Zadeh, MD, PhD, MPH

Harold Simmons Center for Kidney Disease Research and Epidemiology Division of Nephrology and Hypertension, UC Irvine Medical Center 101 The City Drive, Orange, CA 92868 (USA)

E-Mail kkz@uci.edu 
uted to cardiovascular (CV) disease [1]. Despite this enormous CV disease burden and mortality, the main contributors to this risk have not been clearly identified. In fact, traditional risk factors cannot fully explain the magnitude of the risk observed in patients with ESRD and conventional CV risk factors such as hypercholesterolemia and obesity are paradoxically associated with better survival in hemodialysis populations [2-6]. Therefore, while in the general population therapeutic strategies have focused on lowering LDL cholesterol, primarily by the use of HMGCoA reductase inhibitors, this strategy has not been fruitful in patients with ESRD as indicated by the results of 4D, AURORA and SHARP trials [7-9]. Consequently, future studies will need to delineate the association of serum lipids with mortality in patients with ESRD before targeting these indices for therapy. Furthermore, the impact of race and ethnicity on these associations will need to be fully elucidated so that our treatment strategies can be more accurately individualized. The latter is important for several reasons. There is mounting evidence that abnormal lipid profiles differ between racial groups with Caucasians exhibiting higher LDL cholesterol and triglyceride levels than non-Caucasian populations [10]. In addition, the relationship between dyslipidemia and CV disease may differ between racial and ethnic groups. This is demonstrated by a study that showed that smaller LDL particle size is associated with hypertension and coronary heart disease in Caucasians but not in African-Americans [11]. Furthermore, total- and LDL cholesterol concentrations are more strongly associated with $\mathrm{CV}$ disease risk in Caucasians than in African-Americans [12]. These racial and ethnic differences are also important in the ESRD population as we have previously reported that elevated serum LDL cholesterol levels were associated with increased mortality in African-American patients on maintenance hemodialysis. This was in contrast to nonHispanic Caucasians in whom higher LDL levels were associated with improved survival [13].

Most epidemiologic studies examining the role of race and ethnicity divide the population into large cohorts under the categories of Caucasian, African-American, Hispanic and Asian. However, significant heterogeneity can exist within these categories based on each individual's genetic background, culture and country of origin. For example, while epidemiologic studies often regard 'Hispanics' in the United States as a homogenous group, this population has a complex structure comprised of many overlapping subgroups that vary significantly in genetic, environmental and cultural background [14-17]. A generally recognized distinction that has been deciphered from genetic analyses is between Hispanics with primarily Caucasian and African ancestry and those having predominantly Caucasian and Native American ancestry [18-20], with little admixture between individuals of predominantly African versus Native American ancestry. The latter was made possible through the analysis of samples from the multi-ethnic study of atherosclerosis (MESA), which provides one of the most thoroughly characterized samples of Hispanic individuals in the United States. Work using samples from the MESA Hispanic cohort and 199 ancestry informative markers (AIMs) to estimate proportions of ancestry detected significant differences in proportions of European, Native American, and African ancestry by selfidentified country/region of origin. In this study, Mexican/ Central Americans had the highest proportions of Native American ancestry while Puerto Ricans had the highest European ancestry and Dominicans the highest African ancestry [19]. Recent studies have also documented diversity and population substructure within the Native American founder populations [21]. Given these important distinctions, this study was designed to examine the association of lipid profile and CV mortality and all-cause mortality in Hispanic patients on maintenance hemodialysis based on their geographic location. The rationale for this approach is based on the observation that the Hispanic population of the West Coast states (such as California, Arizona and Texas) consists mainly of individuals from Mexico and Central America with predominantly Native American ancestry, while on the East Coast there is a large Afro-Caribbean contingency. Furthermore, the coastal distinctions mentioned here have been used successfully in the past by other investigators to look at genetic variations within the Hispanic population using zip codes as a means of separating this population in two distinct groups [22]. We hypothesized that major differences existed in association of dyslipidemia and survival in Hispanic MHD patients based on their geographic location of residence and hence genetic and ancestral background.

\section{Subjects and Methods}

\section{Study Population}

We extracted, refined, and analyzed data from all individuals with CKD stage 5 who underwent hemodialysis treatments from June 2005 to June 2007 in one of the DaVita Inc., outpatient dialysis facilities. The study was approved by the Institutional Review Committees of the Los Angeles Biomedical Research Institute at Harbor-UCLA and DaVita Clinical Research. Given the large sample size, anonymity of the patients studied, and nonintrusive nature of the research, the requirement for written consent was exempted. 
The first (baseline) studied quarter for each patient was the calendar quarter in which the patient's dialysis vintage was $\geq 90$ days and HDL data was available. Patients who were $\geq 18$ years old, who received hemodialysis in the baseline quarter, and who had available HDL data, were included in the study.

\section{Race/Ethnicity, Other Demographic, and Comorbidity}

Measures

Creation of the cohort has previously been described [23]. Information on race/ethnicity, primary insurance, marital status, and the presence of diabetes at baseline were obtained from the $\mathrm{DaVita}$ database. In the DaVita database, race/ethnicity is self-categorized and patients select the race and/or ethnicity with which they most closely identified according to US Census Bureau [1]. We examined the survival impact of serum lipids in a cohort of 5,185 maintenance hemodialysis patients of Hispanic origin being treated in clinics of a large dialysis organization in California, Texas, New York, New Jersey and Florida.

To minimize measurement variability, all repeated laboratory and clinical measurements for each patient during any given 13week calendar quarter interval were averaged to create a summary estimate that was used in all models. Values were obtained for the baseline quarter (from June 1, 2005 through June 30, 2007) for each patient, and patients' outcomes were followed for 4 additional quarters up to June 30, 2007. Dialysis vintage was defined as the duration of time between the first day of dialysis treatment and the day that the patient entered the cohort study. In addition to laboratory values, post-hemodialysis dry weight and baseline height were used to calculate body mass index (BMI). Data on baseline comorbidities, active tobacco smoking, drug and alcohol dependence were obtained by linking the DaVita database to data from the US Renal Data System (USRDS) Medical Evidence Form 2728. Baseline comorbidities include and were categorized into 11 conditions: atherosclerotic heart disease (AHD), cardiac failure, hypertension, cerebrovascular disease, other cardiac disease, peripheral vascular disease (PVD), chronic obstructive pulmonary disease, malignancy, HIV, AIDS, and non-ambulatory state.

\section{Laboratory Measures}

Blood samples were drawn using standardized techniques in all DaVita dialysis clinics and were transported to the DaVita Laboratory in Deland, Flo., USA, typically within $24 \mathrm{~h}$. All laboratory values were measured using automated and standardized methods in the DaVita laboratory. Most laboratory parameters were measured monthly. Serum ferritin and intact parathyroid hormone were measured at least quarterly. Lipid panels were measured quarterly to annually in select dialysis facilities and in at least $50 \%$ of all outpatients of the clinic during a given calendar quarter. Most blood samples were collected before dialysis, except for serum urea nitrogen that was collected post-dialysis to calculate urea kinetics.

\section{Outcome Ascertainment}

The primary and secondary outcomes of interest were time to all-cause death and CV death, respectively, which were ascertained from the DaVita database as well as through linkage to the USRDS database. CV death data were obtained by identifying the primary cause of death from USRDS. For mortality analyses, patients remained at-risk until death, censoring for renal transplantation, or end of the study period (June 30, 2007).

\section{Statistical Methods}

Patient's baseline demographics, clinical, and laboratory characteristics among each restriction step in the cohort construction were presented as means (standard deviation) and proportions where appropriate. Hispanic patients with cholesterol measured were compared between East and West region using t-test or chisquare where appropriate. Using Cox proportional-hazards regression models, we examined the relation of all-cause mortality or $\mathrm{CV}$ mortality to categories of time-averaged serum cholesterol $(<100,100-<120,120-<140,140-<160,160-<180,180-<200$, $200+\mathrm{mg} / \mathrm{dl}), \mathrm{LDL}(<40,40-<70,70-<100,100-<130,130+\mathrm{mg} / \mathrm{dl})$, HDL $(<30,30-<40,40-<50,50-<60,60+\mathrm{mg} / \mathrm{dl})$ and triglycerides $(<40,40-<70,70-<100,100-<130,130+\mathrm{mg} / \mathrm{dl})$ measurements. For each analysis, three models were examined based on the level of multivariate adjustment: (a) a minimally adjusted model that included mortality as the outcome, lipids, and entry calendar quarter as covariates, (b) case-mix adjusted models that included all of the above plus age, gender, race, diabetes mellitus, dialysis vintage, primary insurance, marital status dialysis dose, and residual renal function during the baseline quarter, the 11 baseline comorbid conditions, active tobacco smoking, drug and alcohol dependence, and (c) malnutrition-inflammation-complex syndrome (MICS) adjusted models, which included all of the covariates in the casemix model as well as 10 surrogates of nutritional status and inflammation: (1) serum albumin, (2) serum TIBC, (3) serum ferritin, (4) serum phosphorus, (5) serum calcium, (6) serum bicarbonate, (7) peripheral white blood cell count (WBC), (8) lymphocyte percentage, (9) hemoglobin, and (10) nPCR as an indicator of daily protein intake, also known as the normalized protein nitrogen appearance (nPNA) [24].

Missing covariate data (under $1 \%$ for most laboratory and demographic variables) were imputed by multiple imputation methods. Plots of log (-log (survival rate)) against log (survival time) were used to check the proportionality assumption. Most analyses were carried out with SAS version 9.1 (SAS Institute, Inc., Cary, N.C., USA). Fractional polynomial graphs were carried out using Stata version 10.1 (StataCorp, College Station, Tex., USA).

\section{Results}

\section{General Data}

The original 2-year national database of all DaVita dialysis patients included 137,260 hemodialysis patients, aged 18 or older and had vintage $\geq 90$ days. After excluding patients who were not Hispanic or living in identified East or West states, the study population consisted of 15,109 patients including 965 Hispanic East and 4,220 Hispanic West patients with cholesterol measured (fig. 1).

Tables 1 and 2 show baseline demographic, clinical, and laboratory characteristics of the main cohort as well as the subset of patients with serum cholesterol measurements. As expected, MHD patients have a higher incidence of low HDL cholesterol (HDL $<40 \mathrm{mg} / \mathrm{dl}$ ) when compared with the general population [25]. In addition, 
Fig. 1. Selection process of the study population.

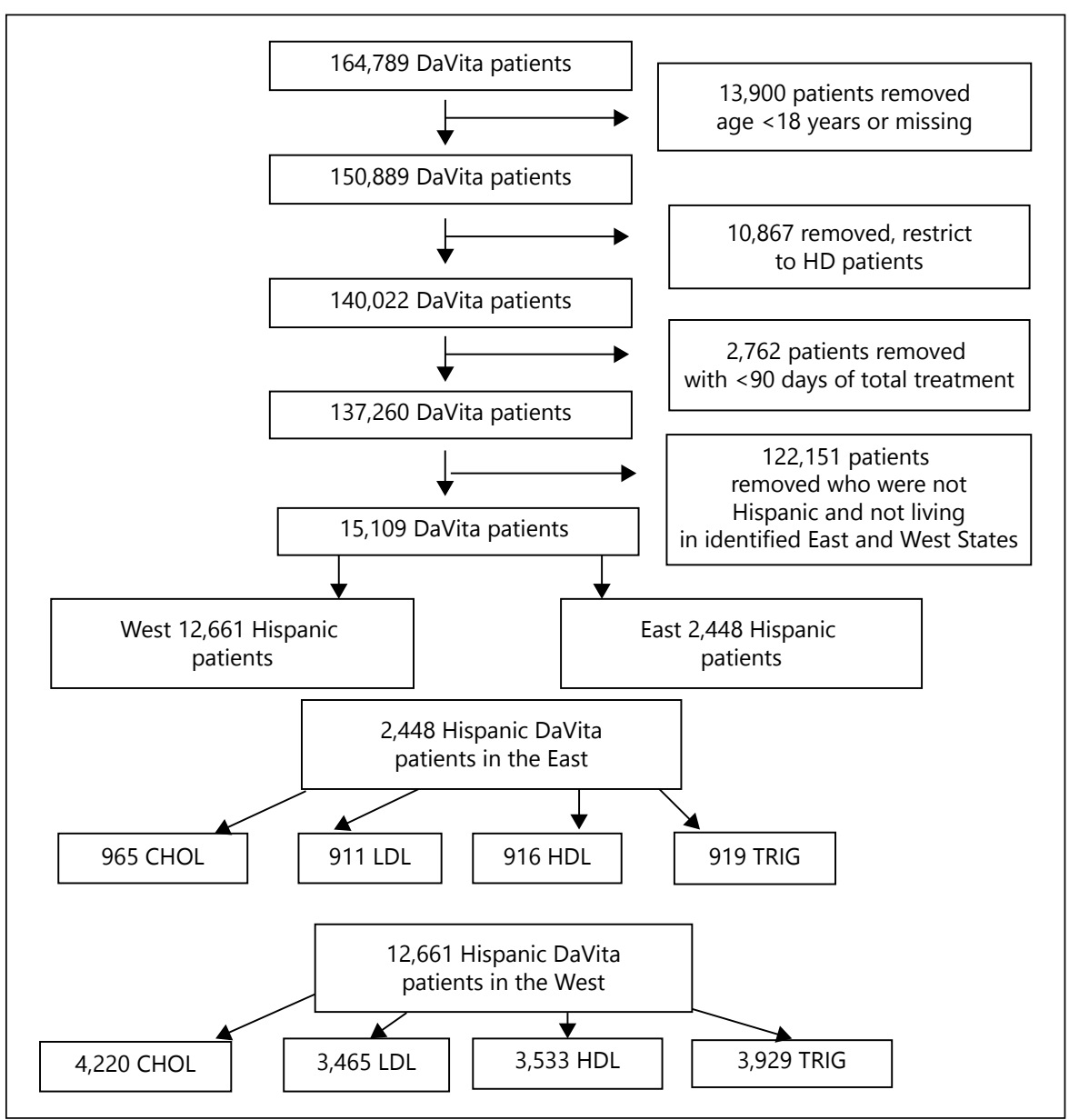

in accordance with our previous studies, serum total and LDL cholesterol levels were not significantly elevated in this patient cohort [13].

\section{Serum Total Cholesterol Level and Mortality}

While the association of serum total cholesterol with all-cause mortality and CV mortality did not reach statistical significance in patients from the West Coast, there did seem to be a trend toward a U-shaped relationship. Hence levels below 100 and above $200 \mathrm{mg} / \mathrm{dl}$ were associated with a nonsignificant trend toward increased mortality. This was in contrast to Hispanic patients from the East Coast in whom higher serum levels of cholesterol were associated with better outcomes. The latter reached statistical significance for CV mortality in those with serum cholesterol levels between 160 and $<180 \mathrm{mg} / \mathrm{dl}$ and above $200 \mathrm{mg} / \mathrm{dl}$ (fig. 2). Therefore, the association of total cholesterol concentrations with mortality in MHD patients of Hispanic origin differs based on their geographic location.

\section{Serum LDL Level and Mortality}

While elevated LDL cholesterol levels (LDL $\geq 130 \mathrm{mg} /$ dl) are associated with a significant increase in all-cause mortality and CV mortality in Hispanic patients from the West Coast, interestingly there seems to be a trend toward better outcomes with elevated LDL levels in Hispanic patients from the East Coast. There is no clear association between low LDL cholesterol levels and mortality in both groups of patients (fig. 3).

\section{Serum HDL Level and Mortality}

The association of serum HDL cholesterol level with allcause mortality and CV mortality follows a U-shaped pattern in Hispanic patients from the West Coast with levels below 30 and above $60 \mathrm{mg} / \mathrm{dl}$ both associated with increases in all-cause mortality with the latter group reaching statistical significance. However, while there is a trend toward worse outcomes with low HDL levels, elevated HDL cholesterol concentrations are associated with a trend toward better overall survival in Hispanic patients from the East 
Table 1. Demographic and clinical characteristics of 137,260 MHD patients, including 15,109 Hispanic MHD patients

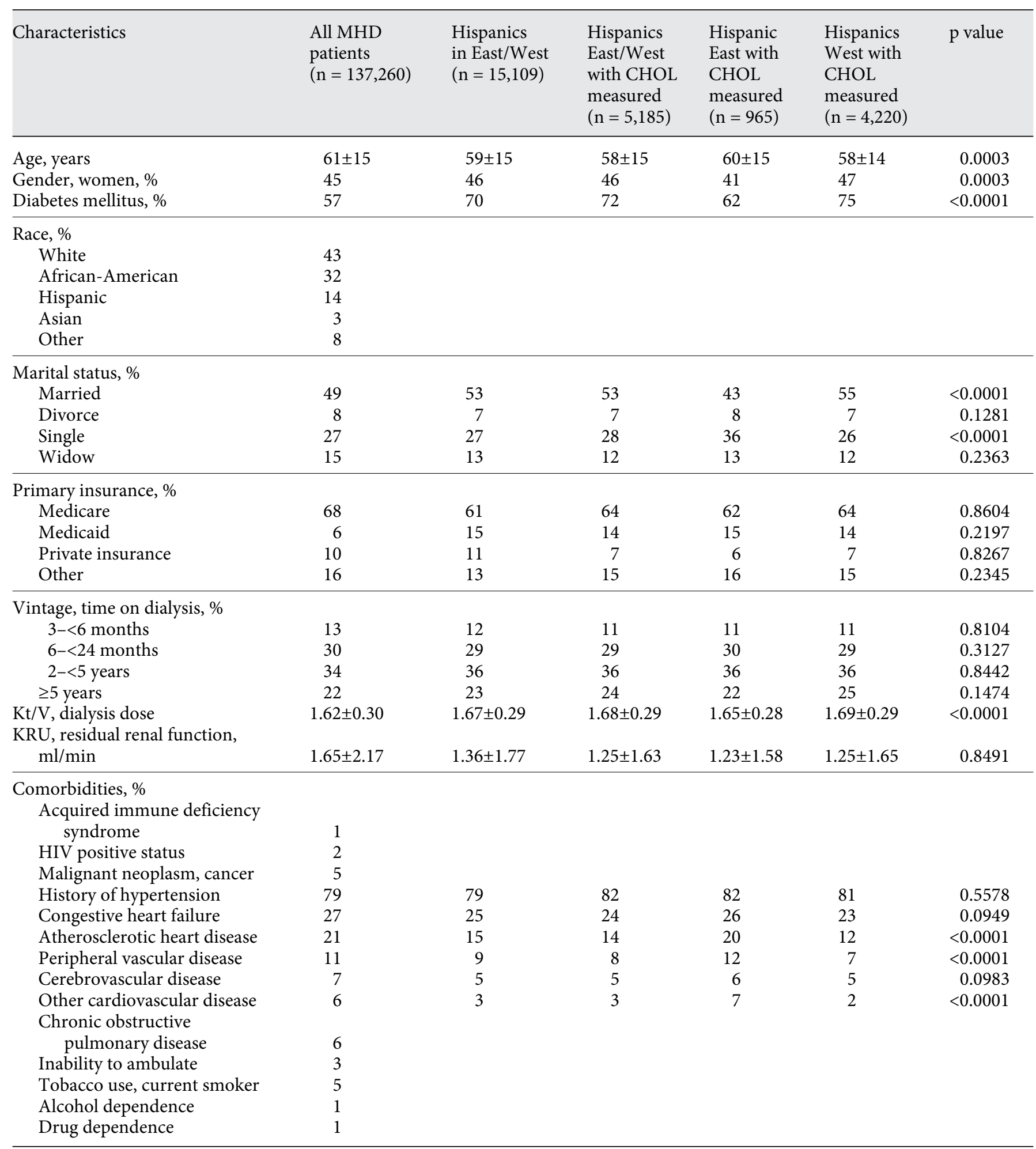

Data presented as mean (SD), or proportions. Comparison between Hispanic East and West with CHOL measurements by t-test for continuous variables, or chi-square test for categorical variables. 
Table 2. Laboratory characteristics of 137,260 MHD patients, including 15,109 MHD patients who had available HDL measurements

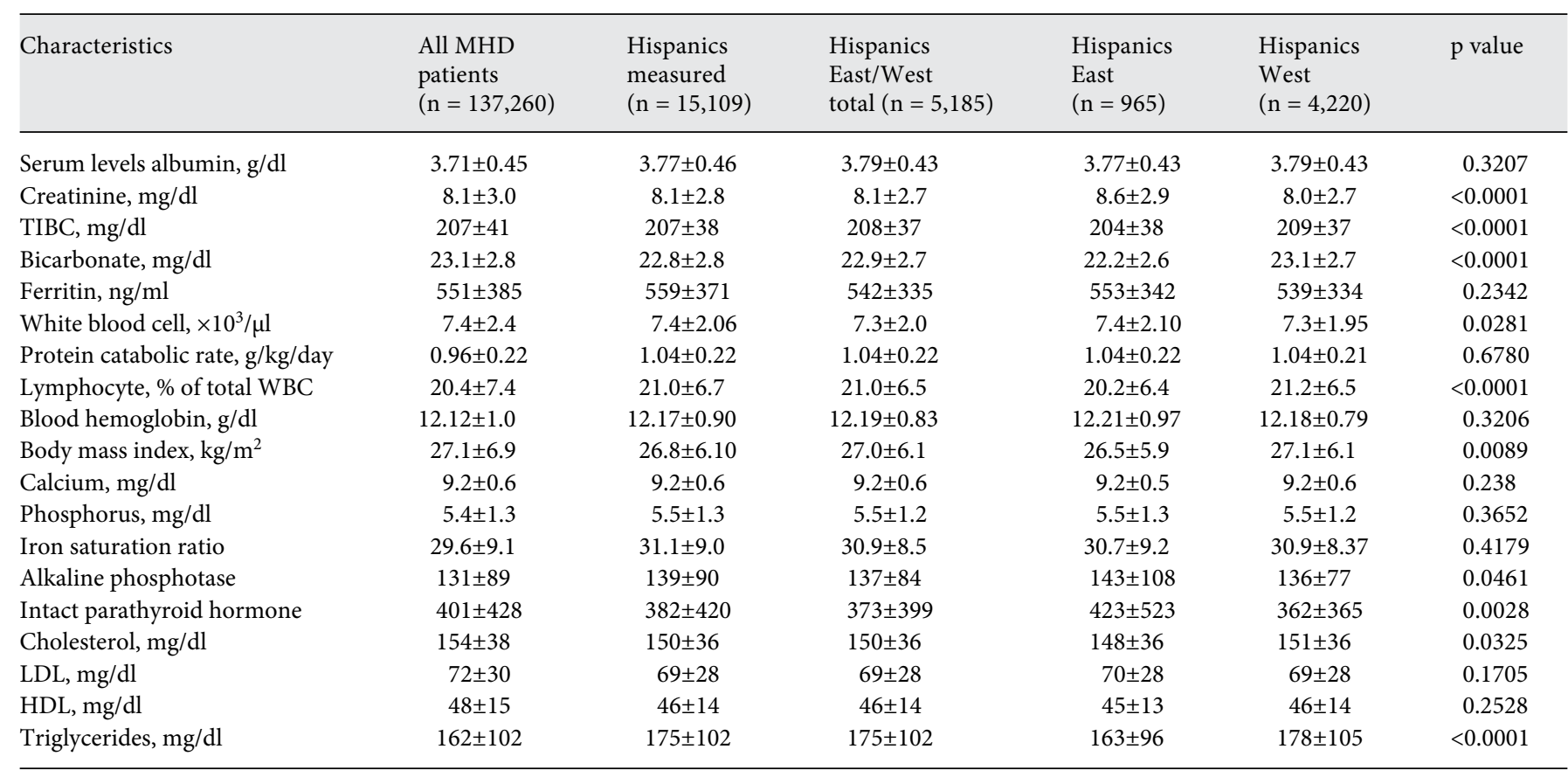

Data presented as mean (SD). Comparison between Hispanic East and West with CHOL measurements by t-test for continuous variables, or chi-square test for categorical variables.

Coast (fig. 4). The association of CV mortality with HDL levels is also different in the two groups in that patients on the East Coast seemed to have a trend toward worse CV outcomes with low HDL, whereas such a trend is not present in Hispanic patients on the West Coast.

\section{Serum Triglyceride Level and Mortality}

While the association between serum triglyceride levels and mortality follows a similar pattern in both Hispanic patients from West and East Coast, notable differences should be pointed out. Low serum triglyceride levels (below 70 $\mathrm{mg} / \mathrm{dl}$ ) are associated with a marked but insignificant increase in mortality in Hispanic patients residing in the West and East Coasts. However, elevated triglyceride levels are associated with a significantly decreased risk of mortality (all-cause and CV) in Hispanic patients from the East Coast, but not in Hispanic patients from the West (fig. 5).

\section{Discussion}

The term 'Hispanic' is arbitrarily applied to an ethnically and racially diverse population of patients who are of Latin American descent. Several studies have docu- mented that the present-day Hispanic population exhibit a complex population structure, with significant genetic variability given contributions from Native American, European as well as African populations [21, 26]. Consequently, these major differences in genetic ancestry together with environmental influences lead to an expression of a variety of phenotypes in different 'Hispanic' groups being studied.

In this study, we divided MHD patients of Hispanic origin based on their geographic area of residence. This division is based on US census data demonstrating that the Hispanic population residing on the East Coast of the United States is mainly made up of individuals with European and African ancestry (i.e. Dominicans, Puerto Ricans and Cubans). This is in contrast to the Hispanic population residing on the West Coast who mainly consists of Native American, Amerindian and European ancestry. This strategy has been utilized previously by other investigators examining admixture proportions of the US Hispanic population [22]. We found that in a large cohort of thrice-weekly treated Hispanic hemodialysis patients followed at a US-based dialysis organization for up to 2 years, significant differences in the association of lipid profiles and mortality existed depending on whether pa- 


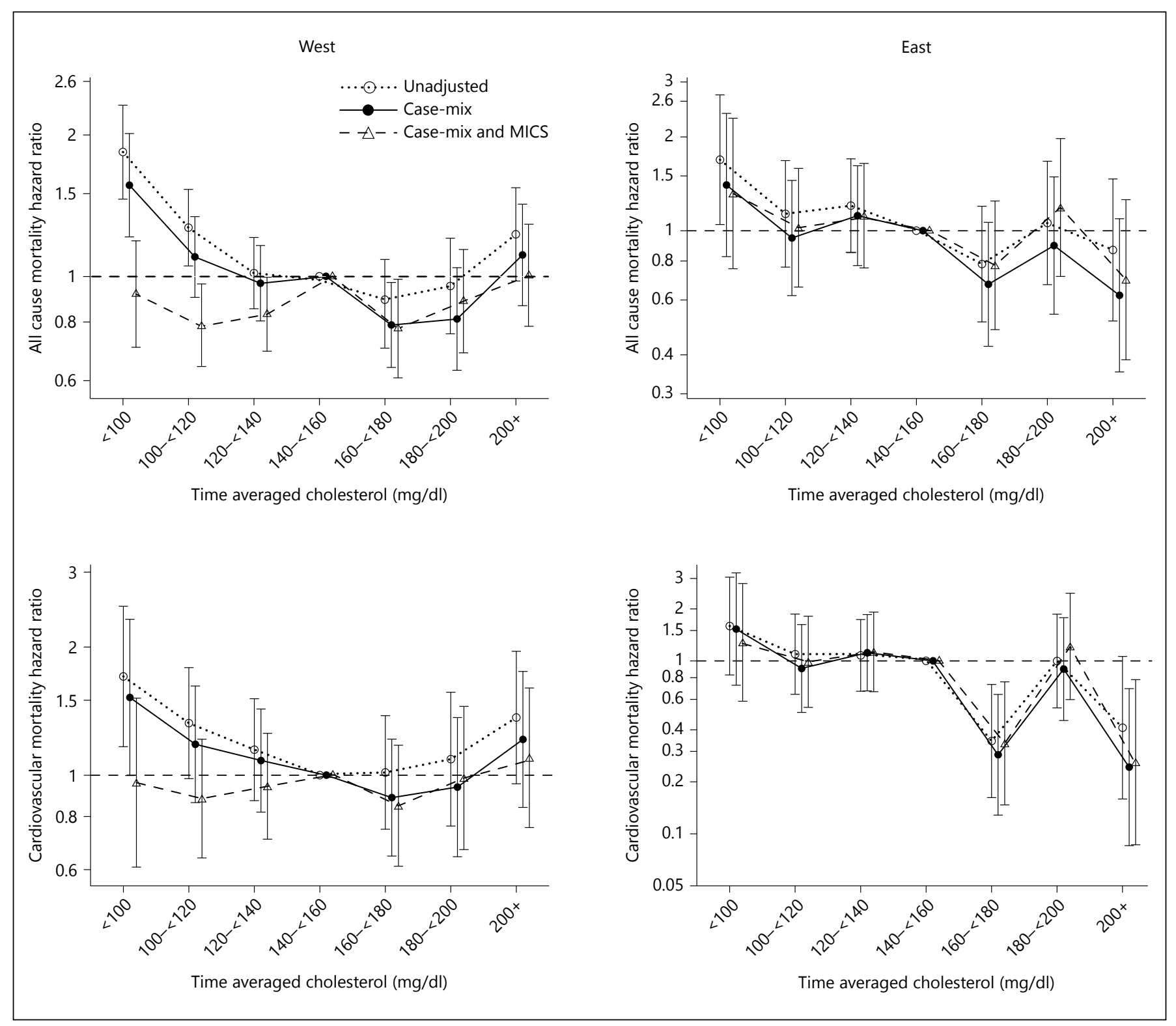

Fig. 2. Unadjusted and adjusted hazard ratios and $95 \%$ confidence intervals for all-cause mortality and CV mortality associated with serum total cholesterol. The model was adjusted for case-mix and markers of malnutrition-inflammation-complex syndrome (MICS).

tients resided in the East versus West Coast. The association of serum total and HDL cholesterol with mortality followed a U-shaped pattern in Hispanic patients residing in the West. This was in contrast to Hispanic patients residing in the East Coast whose survival seemed to improve with increasing total and HDL cholesterol levels. Elevated serum LDL cholesterol levels in Hispanic patients on the West Coast were associated with a significant increase in all-cause mortality, while this association was not ob- served in Hispanic patients residing on the East Coast. Serum triglyceride levels followed a similar trend in both subgroups of Hispanic patients; however, low triglyceride levels were associated with a significant decrease in survival in patients on the West Coast, whereas elevated triglyceride levels were associated with significantly improved survival only in patients residing on the East Coast.

The differences revealed in this study have genetic and biologic plausibility given the findings from several re- 


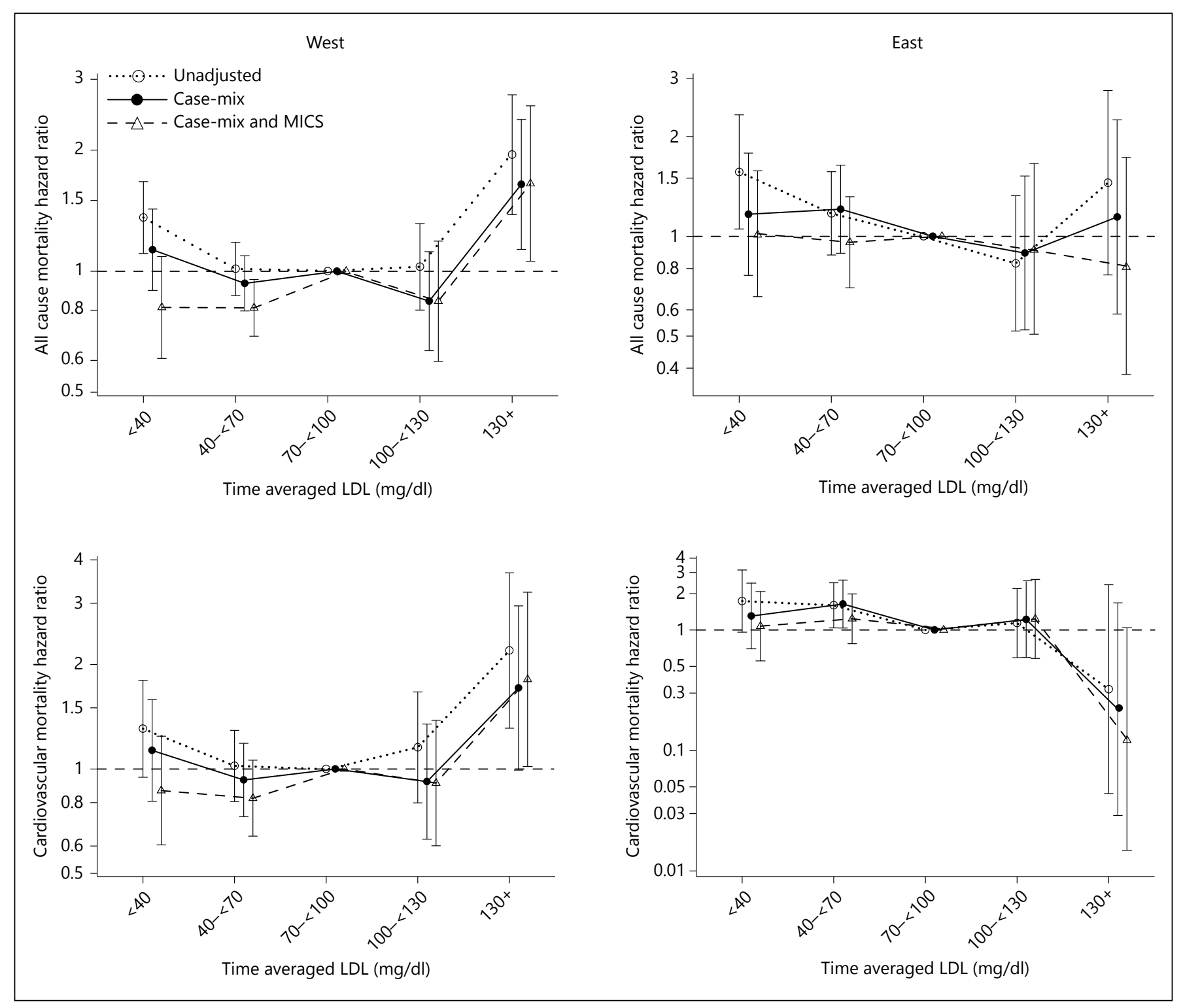

Fig. 3. Unadjusted and adjusted hazard ratios and $95 \%$ confidence intervals for all-cause mortality and CV mortality associated with serum LDL cholesterol. The model was adjusted for case-mix and markers of malnutritioninflammation-complex syndrome (MICS).

cent studies in the Hispanic population, which demonstrated distinct polymorphisms in genes involved in lipid metabolism depending on the cultural and ancestral background of the patients [27-30], for example, using population structure analysis of 1,374 unrelated Hispanic individuals from the Multi-Ethnic Study of Atherosclerosis (MESA).

Manichaikul et al. identified at least four genetically distinct groups within the so-called Hispanic population: Central/South America, the Dominican Republic and
Cuba, Mexico, and Puerto Rico. These differences will be unaccounted for in studies that group this complex population under one category. With regard to lipids, an association analysis of triglycerides was performed with single nucleotide polymorphisms (SNPs) in the lipoprotein lipase (LPL) and tribble homolog 1 (TRIB1) gene regions and statistically significant evidence for genetic heterogeneity at TRIB1 SNP rs4351435 was found. Furthermore, evidence for the TRIB1 SNP rs4351435 was substantially stronger in stratified analysis of the Domin- 


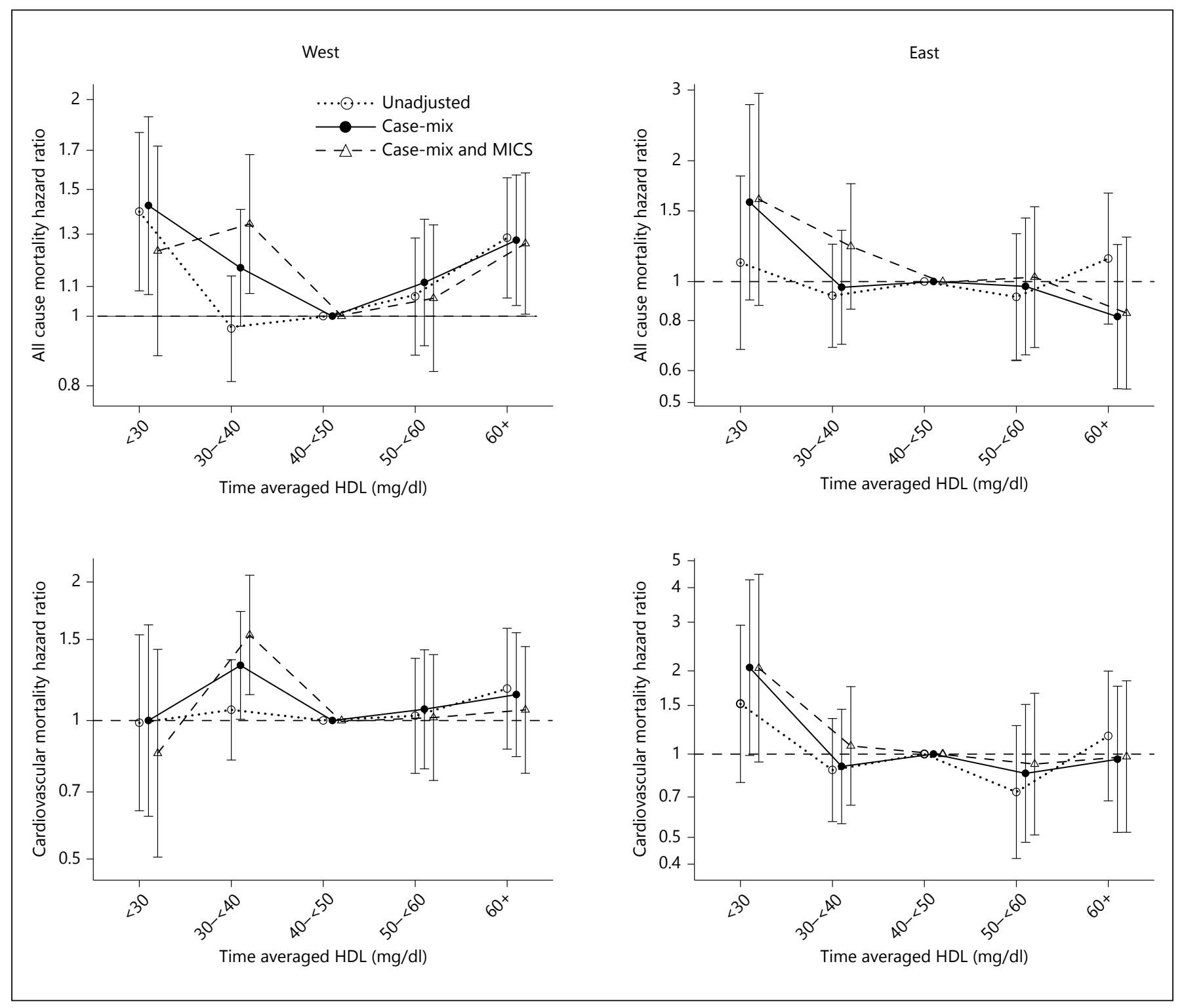

Fig. 4. Unadjusted and adjusted hazard ratios and $95 \%$ confidence intervals for all-cause mortality and CV mortality associated with serum HDL cholesterol. The model was adjusted for case-mix and markers of malnutritioninflammation-complex syndrome (MICS).

ican and Cuban subgroup alone, as compared to pooled analysis of the full MESA Hispanic cohort [30]. Therefore, there may exist other lipid specific genetic polymorphisms within the Hispanic population and this may explain the coastal differences we observed in our patient cohort. In addition, these findings may have significant clinical and therapeutic implications, which can help individualize the treatment of Hispanic patients based on their ethnic and racial background. For example, given that elevated serum LDL cholesterol levels in Hispanic patients on the West are associated with worse outcomes, future studies can target this population of patients for HMG CoA reductase inhibitor therapy.

There were several limitations to our study. First, while the demographic distribution of the US Hispanic population was mostly divided as noted, there may be some overlap of patients between the two coasts. For example, Hispanic patients with Native American ancestry may reside on the East Coast and Hispanic patients with African ancestry may reside on the West Coast. However, our 


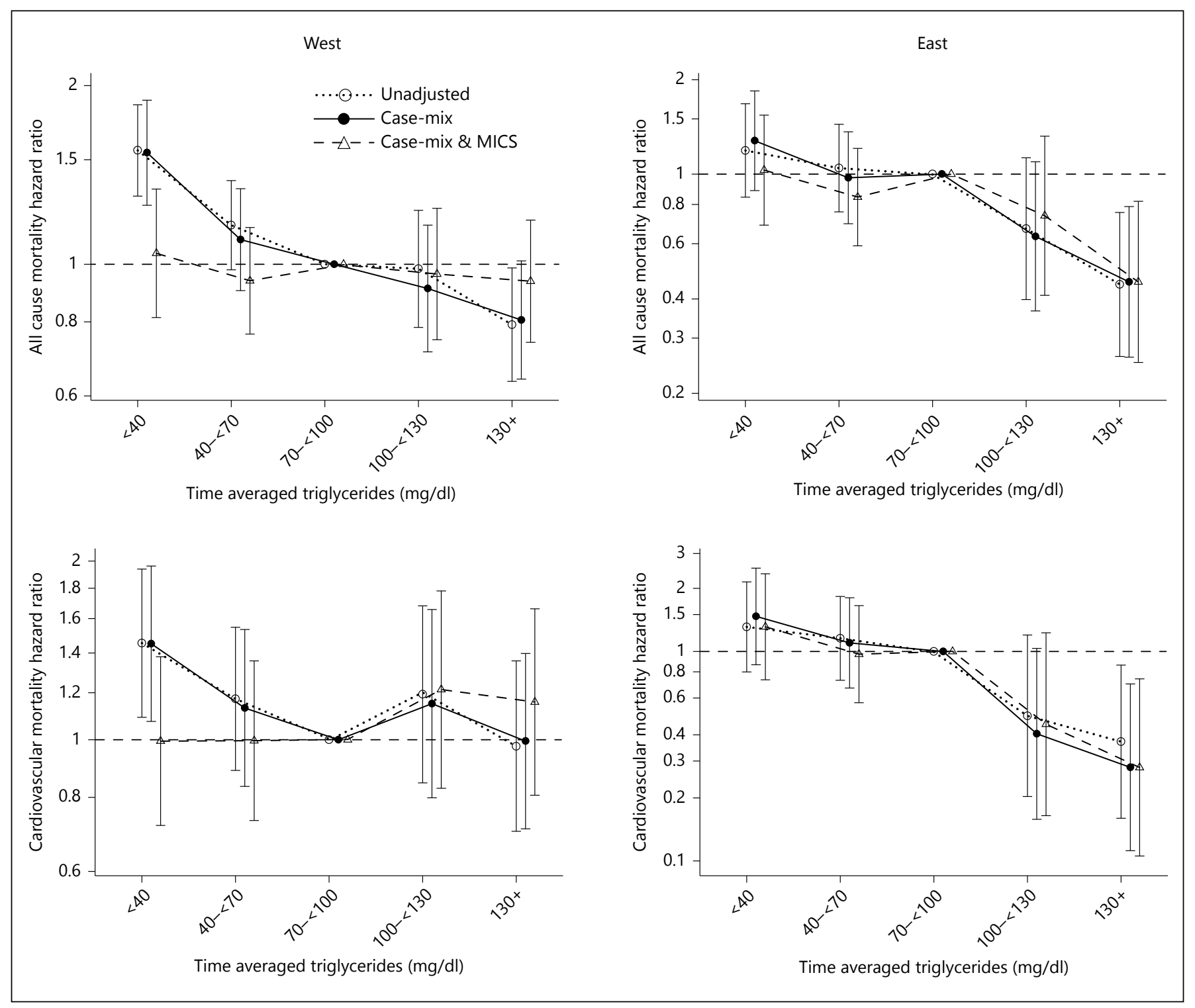

Fig. 5. Unadjusted and adjusted hazard ratios and 95\% confidence intervals for all-cause mortality and CV mortality associated with serum triglyceride cholesterol. The model was adjusted for case-mix and markers of malnutrition-inflammation-complex syndrome (MICS).

study utilized the same methodology as a previous investigation that examined the genetic admixture of this population based on the different coasts. Sensitive genetic analysis in that study found that East Coast Hispanics had higher mean African admixture and the West Coast Hispanics higher mean Indigenous American admixture [22]. Despite this, future studies, which directly evaluate and account for these differences based on data collected directly from the patients, are needed. Second, the current findings should be qualified given the observational na- ture of our study design. In addition, a mixed incident/ prevalent MHD population was examined in this study. However, we did adjust for dialysis vintage in all case-mix models. Another important limitation is the potential confounding of therapy with lipid-altering agents, which was not examined because home medication data were not available systematically in this national cohort. Although lipid-lowering agents may play an important role in modification of mortality risks, to date, most randomized clinical trials of these pharmacologic agents in MHD 
patients have not indicated a survival benefit [7-9]. In addition, there are no studies indicating a geographic difference in prescribing pattern for lipid lowering agents and given that the aim of this study was to compare the East and West Coast Hispanics, and assuming a uniform prescribing pattern between the two coasts, the impact of lipid lowering agents on mortality risk should be spread equally between the two coasts. However, despite the latter points, lack of data on lipid lowering therapy for our cohort is a major limitation of this study and future studies will be needed to account for this important variable. It should also be mentioned that since there is no requirement for fasting prior to collection of predialysis samples, there is no data available on the fasting state of the patients in our cohort. While fasting does not have a major impact on plasma LDL and HDL cholesterol concentrations, serum triglyceride levels have been shown to increase in the post-fed state [31]. Therefore, assuming that our patients were not required to fast, their triglyceride levels may have been altered by predialysis meals. However, there is now emerging data that in apparent contrast to guidelines, several studies have suggested that nonfasting triglycerides may better or similarly predict CV disease events [32-34]. Therefore, many retrospective analyses have been performed using nonfasting lipid panels. Finally, our studied cohort represented only $20 \%$ of the entire national DaVita database and even smaller fractions for subgroup analyses. However, we do not believe confounding by indication was present given that in 2006, the decision to measure serum lipid levels was made uniformly at the clinic level and was not individualized. In addition, we believe the risk of selection bias was not high given that all $\mathrm{DaVita}$ dialysis facilities are under uniform administrative care, and all laboratory tests are performed in one single laboratory with optimal quality-assurance monitoring. Furthermore, we performed a sensitivity analysis to compare DaVita patients included and excluded in this study and did not find any major differences (data not shown).

\section{Conclusions}

Major ethnic and racial differences exist in patients who are grouped as 'Hispanic' in origin. As demonstrated in this study, these differences may have major clinical and therapeutic implications that need to be taken into account. Therefore, future investigations will need to consider these important genetic, racial and ethnic distinctions in order to improve the quality of care being provided and enrich the accuracy of the observations being made in epidemiologic studies.

\section{Acknowledgment}

Some data have been presented in part as abstracts during the American Society of Nephrology (ASN) annual conferences 2013, Atlanta, Ga., USA and ASN 2014, Philadelphia, Pa., USA.

\section{Disclosure Statement}

The authors declare no conflict of interest.

\section{Funding Source}

The study was supported by Dr. Kalantar-Zadeh's research grants from the National Institute of Diabetes, Digestive and Kidney Disease of the National Institute of Health (R01), and a philanthropic grant from Dr. Harold Simmons. Dr. Moradi is supported by Award Number 1IK2CX001043 from the Clinical Research and Development Service of the VA Office of Research and Development.

\section{References}

1 US Renal Data System: USRDS Annual Data Report: Atlas of End-Stage Renal Disease in the United States, 2012.

2 Kalantar-Zadeh K, Kovesdy CP, Derose SF, Horwich TB, Fonarow GC: Racial and survival paradoxes in chronic kidney disease. Nat Clin Pract Nephrol 2007;3:493-506.

3 Kovesdy CP, Anderson JE, Kalantar-Zadeh K: Inverse association between lipid levels and mortality in men with chronic kidney disease who are not yet on dialysis: effects of case mix and the malnutrition-inflammation-cachexia syndrome. J Am Soc Nephrol 2007;18:304-311.

4 Moradi H, Vaziri ND, Kashyap ML, Said HM, Kalantar-Zadeh K: Role of HDL dysfunction in end-stage renal disease: a double-edged sword. J Ren Nutr 2013;23:203-206.

5 Kalantar-Zadeh K, Streja E, Kovesdy CP, Oreopoulos A, Noori N, Jing J, et al: The obesity paradox and mortality associated with surrogates of body size and muscle mass in patients receiving hemodialysis. Mayo Clin Proc 2010;85:991-1001.

6 Molnar MZ, Lukowsky LR, Streja E, Dukkipati R, Jing J, Nissenson AR, et al: Blood pressure and survival in long-term hemodialysis patients with and without polycystic kidney disease. J Hypertens 2010;28:2475-2484.

7 Wanner C, Krane V, März W, Olschewski M, Mann JF, Ruf G, et al; German Diabetes and Di- alysis Study Investigators: Atorvastatin in patients with type 2 diabetes mellitus undergoing hemodialysis. N Engl J Med 2005;353:238-248.

8 Fellström BC, Jardine AG, Schmieder RE, Holdaas $\mathrm{H}$, Bannister $\mathrm{K}$, Beutler $\mathrm{J}$, et al; AURORA Study Group: Rosuvastatin and cardiovascular events in patients undergoing hemodialysis. N Engl J Med 2009;360:1395-1407.

9 Baigent C, Landray MJ, Reith C, Emberson J, Wheeler DC, Tomson C, et al; SHARP Investigators: The effects of lowering LDL cholesterol with simvastatin plus ezetimibe in patients with chronic kidney disease (study of heart and renal protection): a randomised placebo-controlled trial. Lancet 2011;377:2181-2192. 
10 O’Meara JG, Kardia SL, Armon JJ, Brown CA, Boerwinkle E, Turner ST: Ethnic and sex differences in the prevalence, treatment, and control of dyslipidemia among hypertensive adults in the GENOA study. Arch Intern Med 2004;164:1313-1318.

11 Kullo IJ, Jan MF, Bailey KR, Mosley TH, Turner ST: Ethnic differences in low-density lipoprotein particle size in hypertensive adults. J Clin Lipidol 2007;1:218-224.

12 Kral BG, Becker LC, Yook RM, Blumenthal RS, Kwiterovich PO Jr, Otvos JD, et al: Racial differences in low-density lipoprotein particle size in families at high risk for premature coronary heart disease. Ethn Dis 2001;11:325-337.

13 Kilpatrick RD, McAllister CJ, Kovesdy CP, Derose SF, Kopple JD, Kalantar-Zadeh K: Association between serum lipids and survival in hemodialysis patients and impact of race. J Am Soc Nephrol 2007;18:293-303.

14 Choudhry S, Coyle NE, Tang H, Salari K, Lind D, Clark SL, et al: Population stratification confounds genetic association studies among Latinos. Hum Genet 2006;118:652-664.

15 Mao X, Bigham AW, Mei R, Gutierrez G, Weiss KM, Brutsaert TD, et al: A genomewide admixture mapping panel for Hispanic/Latino populations. Am J Hum Genet 2007;80: 1171-1178.

16 Bonilla C, Gutiérrez G, Parra EJ, Kline C, Shriver MD: Admixture analysis of a rural population of the state of Guerrero, Mexico. Am J Phys Anthropol 2005;128:861-869.

17 Bonilla C, Parra EJ, Pfaff CL, Dios S, Marshall JA, Hamman RF, et al: Admixture in the Hispanics of the San Luis Valley, Colorado, and its implications for complex trait gene mapping. Ann Hum Genet 2004;68:139-153.

18 Bryc K, Auton A, Nelson MR, Oksenberg JR, Hauser SL, Williams S, et al: Genome-wide patterns of population structure and admixture in West Africans and African Americans. Proc Natl Acad Sci U S A 2010;107:786-791.

19 Peralta CA, Li Y, Wassel C, Choudhry S, Palmas W, Seldin MF, et al: Differences in albuminuria between Hispanics and Whites: an evaluation by genetic ancestry and country of origin: the multi-ethnic study of atherosclerosis. Circ Cardiovasc Genet 2010;3:240-247.

20 Wang Z, Hildesheim A, Wang SS, Herrero R, Gonzalez P, Burdette L, et al: Genetic admixture and population substructure in Guanacaste Costa Rica. PLoS One 2010;5:e13336.

21 Wang S, Ray N, Rojas W, Parra MV, Bedoya G, Gallo C, et al: Geographic patterns of genome admixture in Latin American Mestizos. PLoS Genet 2008;4:e1000037.

22 Halder I, Yang BZ, Kranzler HR, Stein MB, Shriver MD, Gelernter J: Measurement of admixture proportions and description of admixture structure in different U.S. populations. Hum Mutat 2009;30:1299-1309.

23 Moradi H, Streja E, Kashyap ML, Vaziri ND, Fonarow GC, Kalantar-Zadeh K: Elevated high-density lipoprotein cholesterol and cardiovascular mortality in maintenance hemodialysis patients. Nephrol Dial Transplant 2014;29:1554-1562.

24 Shinaberger CS, Greenland S, Kopple JD, Van Wyck D, Mehrotra R, Kovesdy CP, et al: Is controlling phosphorus by decreasing dietary protein intake beneficial or harmful in persons with chronic kidney disease? Am J Clin Nutr 2008;88:1511-1518.

25 Centers for Disease Control and Prevention NHANES 2009-2010: Laboratory Files HCAfhwcgnnn-lh.

26 Sans M: Admixture studies in Latin America: from the 20th to the 21st century. Hum Biol 2000;72:155-177.
27 Smith CE, Tucker KL, Lee YC, Lai CQ, Parnell LD, Ordovás JM: Low-density lipoprotein receptor-related protein 1 variant interacts with saturated fatty acids in Puerto Ricans. Obesity (Silver Spring) 2013;21:602-608.

28 Coram MA, Duan Q, Hoffmann TJ, Thornton T, Knowles JW, Johnson NA, et al: Genomewide characterization of shared and distinct genetic components that influence blood lipid levels in ethnically diverse human populations. Am J Hum Genet 2013;92:904-916.

29 Frazier-Wood AC, Manichaikul A, Aslibekyan S, Borecki IB, Goff DC, Hopkins PN, et al: Genetic variants associated with VLDL, LDL and HDL particle size differ with race/ethnicity. Hum Genet 2013;132:405-413.

30 Manichaikul A, Palmas W, Rodriguez CJ, Peralta CA, Divers J, Guo X, et al: Population structure of Hispanics in the United States: the multi-ethnic study of atherosclerosis. PLoS Genet 2012;8:e1002640.

31 Rifai N, Cooper GR: Preanalytical variation in lipid, lipoprotein, and apolipoprotein testing; in Rifai N, Dominiczak MH (eds): Handbook of Lipoprotein Testing, ed 2. Washington DC, AAAC Press, 2000, pp 161-187.

32 Bansal S, Buring JE, Rifai N, Mora S, Sacks FM, Ridker PM: Fasting compared with nonfasting triglycerides and risk of cardiovascular events in women. JAMA 2007;298:309-316.

33 Nordestgaard BG, Benn M, Schnohr P, Tybjaerg-Hansen A: Nonfasting triglycerides and risk of myocardial infarction, ischemic heart disease, and death in men and women. JAMA 2007;298:299-308.

34 Eberly LE, Stamler J, Neaton JD; Multiple Risk Factor Intervention Trial Research Group: Relation of triglyceride levels, fasting and nonfasting, to fatal and nonfatal coronary heart disease. Arch Intern Med 2003;163:1077-1083. 